\title{
Fast Implementation of Multiplication on Polynomial Rings
}

\author{
Boyu Wang $(\mathbb{D}$, Haiying Gao $\mathbb{D}$, and Fan Yang $(\mathbb{D}$ \\ PLA SSF Information Engineering University, Zhengzhou, China \\ Correspondence should be addressed to Haiying Gao; xdgaohaiying@163.com
}

Received 12 October 2021; Revised 6 December 2021; Accepted 29 December 2021; Published 14 February 2022

Academic Editor: Iqtadar Hussain

Copyright (c) 2022 Boyu Wang et al. This is an open access article distributed under the Creative Commons Attribution License, which permits unrestricted use, distribution, and reproduction in any medium, provided the original work is properly cited.

Multiplication on polynomial rings has been widely used in public-key cryptographic schemes based on ideal lattices. It is an important module that significantly affects the efficiency of the schemes. Improved Preprocess-then-NTT (IPtNTT) is an algorithm which can fast realize multiplication on polynomial rings. Compared with the Number Theoretic Transform (NTT), the IPtNTT weakens the parameter restriction of lattice-based public-key cryptographic schemes. By optimizing the IPtNTT with the AVX2 instruction set, we reduce the clock cycles consumed by multiplication on polynomial rings to $15 \%-22 \%$. According to the experimental results, we give specific suggestions on using AVX2 optimized IPtNTT to realize multiplication on polynomial rings with different parameters chosen in lattice-based public-key cryptosystems.

\section{Introduction}

Lattice-based public-key cryptography has emerged as a hot research direction in postquantum cryptography. Compared with the classic hard lattice problems such as the shortest vector problem and the closest vector problem, the Learning with Errors (LWE) problem [1] seems more suitable for constructing lattice-based public-key cryptographic schemes, especially the Ring-Learning with Errors (RLWE) problem [2] on which NEWHOPE is based, and the ModuleLearning with Errors (MLWE) problem [3] on which KYBER is based. Multiplication on polynomial rings is a necessary and time-consuming operation in lattice cryptosystems. Through studying its fast implementation, we can significantly improve the multiplication efficiency.

Various algorithms including Karatsuba algorithm [4,5] and Fast Fourier Transform (FFT) algorithm [6] have been proposed to improve the implementation efficiency of polynomial multiplication. If we take the coefficients of the polynomial from the quotient ring $\mathbb{Z}_{q}=\mathbb{Z} / q \mathbb{Z}$, we call FFT the Number Theoretic Transform (NTT) [6]. NTT uses the root of unity in the residue class ring instead of that in the field of complexity number applied in FFT, which improves the calculation accuracy. In a polynomial ring $R_{q}=\mathbb{Z}_{q}[x] /\left(x^{n}+1\right)$, where $q$ is a prime and $n$ is some power of 2, the product of two polynomials corresponds to the cyclic convolution of the coefficient vectors. If $q$ and $n$ satisfy $q \equiv 1 \bmod 2 n$, which means there exist $2 n$-th roots of unity in $\mathbb{Z}_{q}$, we can efficiently multiply polynomials through NTT.

However, the condition that $q$ and $n$ must satisfy to fasten the multiplication on polynomial rings through NTT severely limits the parameter selection in lattice-based public-key cryptosystem. In Inscrypt 2018, Zhou et al. [7] proposed a technique called Preprocess-then-NTT (PtNTT) which divides the $n$-th polynomials into several polynomials of lower degree $\left(n / 2^{r}\right)$-th, where $r$ is a positive integer) and then performs the NTT for these $n / 2^{r}$-th polynomials, respectively. Since $n$ and $q$ are required to satisfy $n / 2^{r-1} \mid(q-1)$, the selection range of $q$ in lattice-based public-key cryptosystem is enlarged. But the calculation efficiency of multiplication through PtNTT is lower than that through the original NTT due to some additional procedures.

In order to solve the efficiency problem, Zhu et al. [8] introduced a technique called Improved Preprocess-thenNTT (IPtNTT). Similar to PtNTT, IPtNTT also divides the polynomials into several polynomials of lower degree and then applies NTT to their multiplications. But in the final procedure, they completed the multiplication using some ideas of Karatsuba algorithm, so as to optimize the implementation efficiency. 
To further improve the implementation efficiency, advanced vector extensions (AVX), a new set of SIMD instructions that are compatible with AMD's SSE5 instruction sets, are often used. The AVX instructions deal with 128-bit wide data. Making better use of registers, the AVX2 instruction set widens the width to 256 bits to improve the computing capability of the processor. Seiler [9] presented a method to optimize NTT with AVX2 instructions to implement NTT more efficiently. However, how to use AVX2 instruction set to accelerate IPtNTT needs further research.

Aiming at accelerating the implementation of IPtNTT algorithm with AVX2 instruction set, we first give the AVX2 implementation methods of modular operation and coefficients shuffling, respectively. We then demonstrate our AVX2 optimization to NTT and $\mathrm{NTT}^{-1}$ transformation on this basis. After deducing the specification of the reduction to the point-wise multiplications in [8], we finally present AVX2 optimized IPtNTT. Having made several experiments with some common parameters used in lattice-based public-key encryption schemes, we compare the implementation efficiency before and after optimization. The experimental results show that the clock cycles consumed by AVX 2 optimized $\alpha$ IPtNTT are $15 \%-22 \%$ of standard $\mathrm{C}$ implementation. According to the experimental results, we give specific suggestions on using AVX2 optimized IPtNTT to realize multiplication on polynomial rings with certain $q$ and $n$.

1.1. Roadmap. The remainder of the paper is organized as follows. In Section 2, we provide preliminaries and notations. In Section 3, we present the AVX2 optimization to IPtNTT. In Section 4, we show the experimental results and the analysis. In Section 5, we give the conclusion.

\section{Preliminaries}

Let $\mathbb{Z}$ be the integer ring, $q$ be a prime, and $\mathbb{Z}_{q}$ represent the residue class ring $\mathbb{Z} / q \mathbb{Z}$, which means $\mathbb{Z}_{q}=\{0, \ldots, q-1\}$. We have $R_{q}=\mathbb{Z}_{q}[x] /\left(x^{n}+1\right)$ which denotes the polynomial ring consisting of polynomials modulo $x^{n}+1$, whose coefficients are chosen from $\mathbb{Z}_{q}$. For an arbitrary element $f$ belonging to $R_{q}$, it can be written as $f=\sum_{i=0}^{n-1} f_{i} x^{i}$, where $f_{i} \in \mathbb{Z}_{q}$. We can also consider $f$ as a vector containing coefficients of the polynomial, which means $f \leftrightarrow\left(f_{n-1}, f_{n-2}, \ldots, f_{0}\right)$. For $f, g \in R_{q}$, we have $f \circ g$ which denotes the point-wise multiplication of $f$ and $g$, which means $f \circ g \leftrightarrow\left(f_{n-1} \cdot g_{n-1} \bmod q, f_{n-2} \cdot g_{n-2} \bmod q, \ldots\right.$, $\left.f_{0} \cdot g_{0} \bmod q\right) . f \cdot g$ represents the product or the cyclic convolution of $f$ and $g$. If $h=f \cdot g \in R_{q}$, then

$$
h_{i}=\left(\sum_{j=0}^{i} f_{j} g_{i-j}-\sum_{j=i+1}^{n-1} f_{j} g_{n+i-j}\right) \bmod q,
$$

and we can see its computational complexity is $T(n)=n^{2}$.

\subsection{Multiplication on Polynomial Rings Based on NTT.} We can fast realize the multiplication on polynomial rings through the forward transformation NTT, the inverse transformation $\mathrm{NTT}^{-1}$, and the point-wise multiplication on $R_{q}$ [10]. We then present the corresponding basic knowledge.
Now, we have an integer $n$ which satisfies $n=2^{m}, m \in \mathbb{Z}$, and a prime $q$ which satisfies $q \equiv 1 \bmod 2 n$. Let $\omega$ be a $n$-th primitive root of unity in $\mathbb{Z}_{q}$ and $\gamma$ be a $2 n$-th primitive root of unity, which means $\gamma=\sqrt{\omega}$. Then for $\forall f \in R_{q}$, we can describe its forward transformation NTT as $\widehat{f}_{i}=\sum_{j=0}^{n-1} \gamma^{j} f_{j} \cdot \omega^{i j} \bmod q$. Note that $\hat{f} \leftrightarrow\left(\hat{f}_{n-1}, \widehat{f}_{n-2}, \ldots, \widehat{f}_{0}\right)$, where $\widehat{f}=\operatorname{NTT}(f)$. Accordingly, for $\forall \hat{f} \in R_{q}$, the inverse transformation $\mathrm{NTT}^{-1}$ can be described as $f_{i}=n^{-1} \sum_{j=0}^{n-1} \gamma^{j} \widehat{f}_{j} \cdot \omega^{-i j} \bmod q, \quad$ which means $f=\operatorname{NTT}^{-1}(\hat{f})$. So, we can compute $f \cdot g$ by

$$
f \cdot g=\mathrm{NTT}^{-1}\left(\mathrm{NTT}(f)^{\circ} \mathrm{NTT}(\mathrm{g})\right) .
$$

If we precompute and store the powers of the root, the computational complexity of NTT or $\mathrm{NTT}^{-1}$ transformation is $T(n)=n^{2}$, and that of equation (2) is $T(n)=3 n^{2}+n$. It is an unacceptable complexity which is even larger than that of equation (1). However, Eleanor et al. [11] proposed a fast implementation to reduce the computational complexity of NTT and $\mathrm{NTT}^{-1}$ transformation to $T(n)=n \log n$, thus reducing that of equation (2) to $T(n)=3 n \log n+n$.

Here, we briefly introduce a method with divide-andconquer strategy to implement NTT fast. Dividing the sequence of polynomial coefficients into two $n / 2$ long subsequences according to the parity of their subscripts, we then have $f_{i}^{\prime}=f_{2 i}$ and $f_{i}^{\prime \prime}=f_{2 i+1}$, where $i=0,1, \ldots, n / 2-1$. Their NTT transformed results turn out to be $\widehat{f}_{i}^{\prime}=\sum_{j=0}^{n / 2-1} \widetilde{\gamma}^{j} \cdot f_{j}^{\prime} \cdot \widetilde{\omega}^{i j} \bmod q$ and $\hat{f}_{i}^{\prime \prime}=\sum_{j=0}^{n / 2-1} \tilde{\gamma}^{j} \cdot f_{j}^{\prime \prime} \cdot \widetilde{\omega}^{i j} \bmod q$, respectively, where $\widetilde{\omega}$ is a $n / 2$-th primitive root of unity in $\mathbb{Z}_{q}$ and $\widetilde{\gamma}$ is a $n$-th root, which means $\widetilde{\gamma}=\omega$. After the transformation in this level, the computational complexity changes from one $n$-th NTT transformation to two $n / 2$-th NTTs, which is $T(n)=n^{2} / 2$. We repeat such transformation level by level until each subsequence contains only 1 coefficient and finally do the NTT to each pair of subsequences. In this way, the computational complexity is $T(n)=n \log n$. Note that the subscripts of NTT transformed coefficients are bitreversed. If $k$ is an integer smaller than $n$ which can be described as $k=k_{m-1} 2^{m-1}+k_{m-2} 2^{m-2}+\cdots+k_{1} 2+k_{0}$ and brv $(k)$ represents the bit-reversed $k$, we have $\operatorname{brv}(k)=k_{0} 2^{m-1}+k_{1} 2^{m-2}+\cdots+k_{m-2} 2+k_{m-1}$. In order to demonstrate the transformation more explicitly, we use $f(x)$ to represent the $x$-th coefficient of $f$ with $x$ shown in binary while being transformed level by level. Let $f_{-i}$ be the transformation result in the $i$-th level and $A(\operatorname{brv}(x))$ be the brv $(x)$-th coefficient in the last level; then, the result of the $m$-th level is $\widehat{f} \leftrightarrow(A(0), A(1), \ldots, A(n-1)) \quad \leftrightarrow\left(f_{-m}(\operatorname{brv}(0)), f_{-m}\right.$ $\left.(\operatorname{brv}(n / 2)), \ldots, f_{-m}(\operatorname{brv}(n-1))\right)$. Therefore, the transformation in the first level can be described as follows:

$$
\begin{aligned}
f_{-1}(i)= & f_{-0}(i)+\gamma^{\operatorname{brv}(1)} f_{-0}(i+\operatorname{brv}(1)), \\
f_{-1}(i+\operatorname{brv}(1))= & f_{-0}(i)-\gamma^{\operatorname{brv}(1)} \\
& \cdot f_{-0}(i+\operatorname{brv}(1)), \quad 0 \leq i \leq \frac{n}{2}-1 .
\end{aligned}
$$

Similarly, we can construct relationships of subsequent levels. Figure 1 shows the structure of the fast NTT 
implementation. By inversely doing the transformation, we get the structure of $\mathrm{NTT}^{-1}$ which shares the same complexity with NTT.

2.2. Improved Preprocess-Then-NTT. In IPtNTT, $f$ and $g$ are divided into several shorter polynomials. After NTT, pointwise multiplication, and $\mathrm{NTT}^{-1}$, the coefficients of those shorter polynomials turn to be a series of coefficient vectors $\bar{h}_{i}$, which are finally combined into the coefficient vector corresponding to $f \cdot g$. Algorithm 1 is the $\alpha$-round IPtNTT $(\alpha$ IPtNTT) algorithm proposed by Zhu et al. [8], which requires $q \equiv 1\left(\bmod n / 2^{\alpha-1}\right)$. In Algorithm $1, z$ represents $x^{2^{\alpha}}$, where $\alpha \geq 1$, and $\operatorname{NTT}(z)$ can be precomputed. In Step 2, when they compute each part, for $i=j$, they do it as usual, while for $i \neq j$, they compute two corresponding parts together as follows in practice:

$$
\begin{aligned}
& \operatorname{NTT}\left(\bar{f}_{i}\right)^{\circ} \operatorname{NTT}\left(\overline{\mathrm{g}}_{\mathrm{j}}\right)+\operatorname{NTT}\left(\overline{\mathrm{f}}_{\mathrm{j}}\right) \circ \operatorname{NTT}\left(\overline{\mathrm{g}}_{\mathrm{i}}\right) \\
= & \left(\operatorname{NTT}\left(\bar{f}_{i}\right)+\operatorname{NTT}\left(\bar{f}_{\mathrm{j}}\right)\right) \circ\left(\operatorname{NTT}\left(\overline{\mathrm{g}}_{\mathrm{i}}\right)+\operatorname{NTT}\left(\overline{\mathrm{g}}_{\mathrm{j}}\right)\right)-\operatorname{NTT}\left(\overline{\mathrm{f}}_{\mathrm{i}}\right) \circ \operatorname{NTT}\left(\overline{\mathrm{g}}_{\mathrm{i}}\right)-\operatorname{NTT}\left(\overline{\mathrm{f}}_{\mathrm{j}}\right) \circ \operatorname{NTT}\left(\overline{\mathrm{g}}_{\mathrm{j}}\right) .
\end{aligned}
$$

In this way, they reduce the point-wise multiplications when $i \neq j$. Throughout this paper, we use $\widehat{*}$ to represent $\operatorname{NTT}(*)$.

Their result shows that $\alpha \mathrm{IPtNTT}$ is composed of $n / 2^{\alpha}$-th NTTs, $n / 2^{\alpha}$-th $\mathrm{NTT}^{-1}$ s, and $n / 2^{\alpha}$-th point-wise multiplications, and the number of them is $2^{\alpha+1}, 2^{\alpha}$ and $3 \cdot 2^{2 \alpha-2}+2^{\alpha-1}$, respectively. So, the total computational complexity is as follows:

$$
T(n)=3 n \log n+\left(3 \cdot 2^{\alpha-2}-3 \alpha+\frac{1}{2}\right) n .
$$

\section{AVX2 Optimization to IPtNTT}

Taking advantage of its parallel computation, we use AVX2 instructions to optimize IPtNTT. As the parameter qs commonly chosen by LWE cryptosystems like 3329, 7681, 12289 or the even smaller one 1409 are all within 16 bits, we determine to store 16 numbers in 1 register. So, each AVX2 instruction deals with vectors containing 16 numbers. According to Algorithm 1, we find that the key events to optimize $\alpha$ IPtNTT include AVX2 optimized modular reduction, coefficients shuffling, NTT, $\mathrm{NTT}^{-1}$, and point-wise multiplication. The logical relationship is shown in Figure 2. We introduce our implementation, respectively, in the following.

3.1. AVX2 Implemented Modular Reduction. As there exists no instruction to acquire remainders, we implement the modified Montgomery transformation and the modified Barrett transformation proposed by Seiler [9] which are called Montgomery transformation and Barrett transformation, respectively, throughout this paper. Specifically, Montgomery transformation deals with numbers within 32 bits, while Barrett transformation deals with those within 16 bits. This subsection presents how we implement such transformations with AVX2 instructions.

As for the specific realization of the algorithm, we move the product of two 16-bit numbers to the right for 16 bits by using the instruction which gets the high 16 bits of the product. The instruction to acquire low 16 bits can efficiently get the remainder of the product modulo $2^{16}$. In Barrett transformation, a number that can be precomputed needs to be rounded, which can be achieved by adding 0.5 to the original number and then rounding it down. The specific implementation procedure is shown in Algorithms 2 and 3.

3.2. AVX2 Implemented Shuffling. According to the description in 2.1, the fast implementation of NTT is hierarchical. In the forward transformation NTT, for each level before the fourth level from the bottom, all sixteen coefficients in one vector use the same power of the chosen root. So, we can use _mm256_loadu_si256 to take out the two coefficient vectors and directly do the computation according to the relationship of each two levels similar to equation (3). In the last 4 levels, after taken out by_mm256_loadu_si256, coefficients in the two vectors need different powers of the root to finish the transformation, but the two coefficients in one relation stored in the same vector. Therefore, it is necessary to shuffle the elements of the coefficient vectors.

In the fourth level from the bottom in NTT transformation, eight coefficients in one vector are performed with the other eight using the same root. We need to switch the lower eight in the vector containing higher subscript coefficients with the higher eight in the lower vector. We first use _mm256_permute4x64_epi64 (*,0xD8) to both vectors and then get the coefficients with higher subscripts in those relations with_mm256_unpackhi_epi64 $(*, * *)$ and the lowers with_mm256_unpacklo_epi64 (*,**). After we use _mm256_permute4x64_epi64 $\left({ }^{*}, 0 \mathrm{xD} 8\right)$ to both vectors again, the two corresponding coefficients in relations similar to equation (3) are at the corresponding positions of the two vectors. We present the process of coefficients shuffling in the fourth level from the bottom of the forward transformation in Figure 3. Notice that there is no need to restore the coefficients to the original order for the next level after NTT. We can do above shuffles the other way around in the first four levels in $\mathrm{NTT}^{-1}$. Shuffles in other levels can be performed similarly.

3.3. A VX2 Implementation of NTTand NTT ${ }^{-1}$. As mentioned above, the fast implementation of NTT relies on hierarchical operations, each of which involves coefficients' modulo multiplication with the corresponding powers of the root of unity. Since we use Montgomery transformation in our AVX2 implementation, what we actually get is $2^{-16} a(\bmod q)$, where $a$ is our target. Therefore, when we build the table of the 


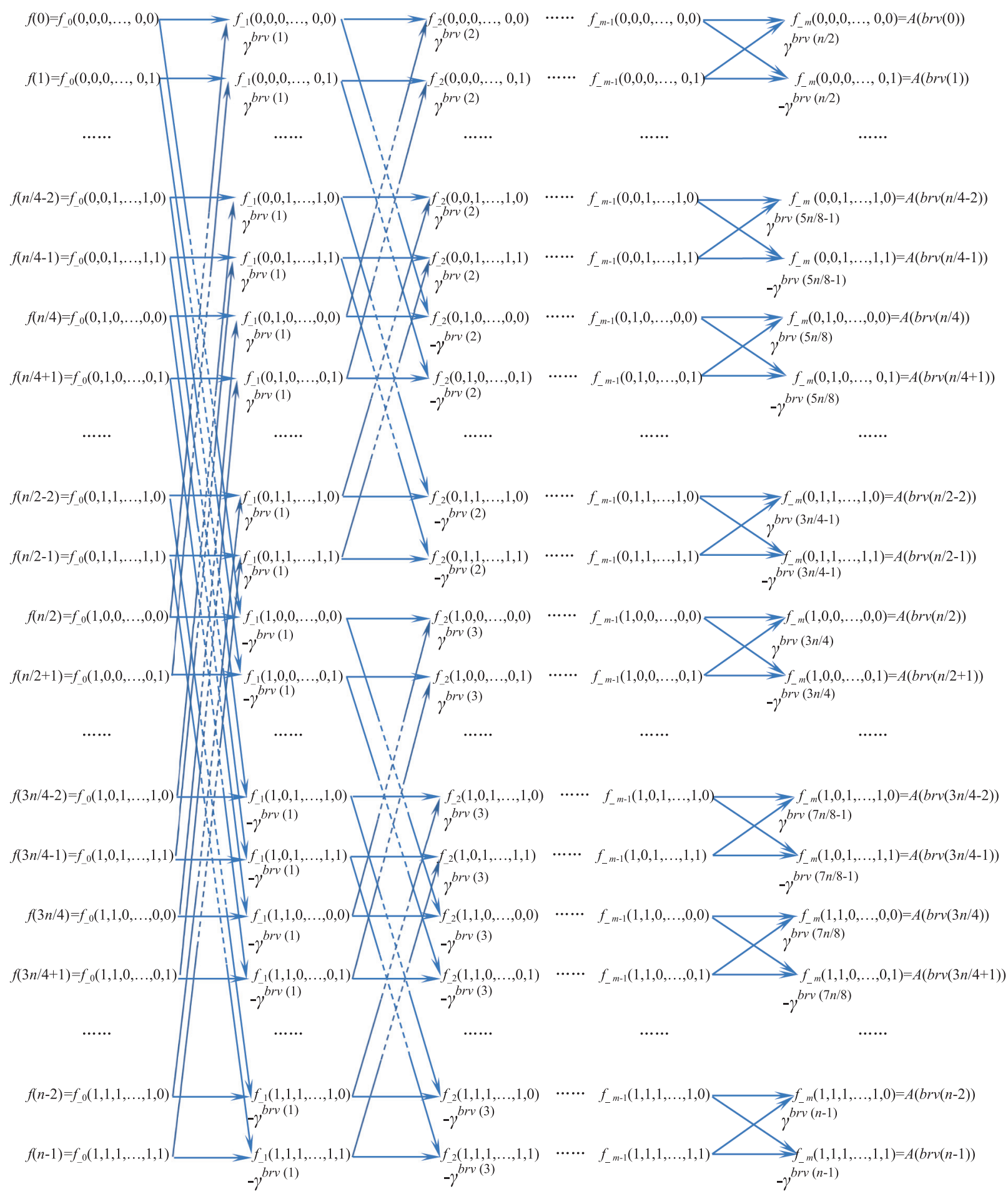

FIGURE 1: Structure of fast NTT implementation.

Input: coefficient vectors of $f$ and $g$

Output: coefficient vector of $h=f \cdot g$

Step 1: (Separation) $f(x)=\sum_{i=0}^{2^{\alpha}-1} x^{i} \cdot \bar{f}_{i}\left(x^{2^{\alpha}}\right), g(x)=\sum_{j=0}^{2^{\alpha}-1} x^{j} \cdot \bar{g}_{j}\left(x^{2^{\alpha}}\right)$, where $\bar{f}_{i}(z), \bar{g}_{j}(z)$ are all $n / 2^{\alpha}$-th.

Step 2: (Multiplication) Compute $\bar{h}_{i}(z) \in \mathbb{Z}_{q}[z] /\left(z^{n / 2^{\alpha}}+1\right)$ as follows: $\bar{h}_{i}(z)=\sum_{l=0}^{i} \bar{f}_{l}(z) \cdot \bar{g}_{i-l}(z)+\sum_{l=i+1}^{2^{\alpha}-1} z$. $\bar{f}_{l}(z) \cdot \bar{g}_{2^{\alpha}+i-l}(z)=\operatorname{NTT}^{-1}\left(\sum_{l=0}^{i} \operatorname{NTT}\left(\bar{f}_{l}(z)\right)^{\circ} \operatorname{NTT}\left(\overline{\mathrm{g}}_{\mathrm{i}-1}(\mathrm{z})\right)+\operatorname{NTT}(z)^{\circ} \sum_{\mathrm{l}=\mathrm{i}+1}^{2^{\alpha}} \operatorname{NTT}\left(\overline{\mathrm{f}}_{1}(\mathrm{z})\right)^{\circ} \mathrm{NTT}\left(\overline{\mathrm{g}}_{2^{\alpha}+\mathrm{i}-1}(\mathrm{z})\right)\right)$, where $0 \leq i \leq 2^{\alpha}$.

Step 3: (Gatheration) Compute $h$ in $R_{q}$ as follows: $h(x)=\sum_{i=0}^{2^{\alpha}-1} x^{i} \cdot \bar{h}_{i}\left(x^{2^{\alpha}}\right)$. 


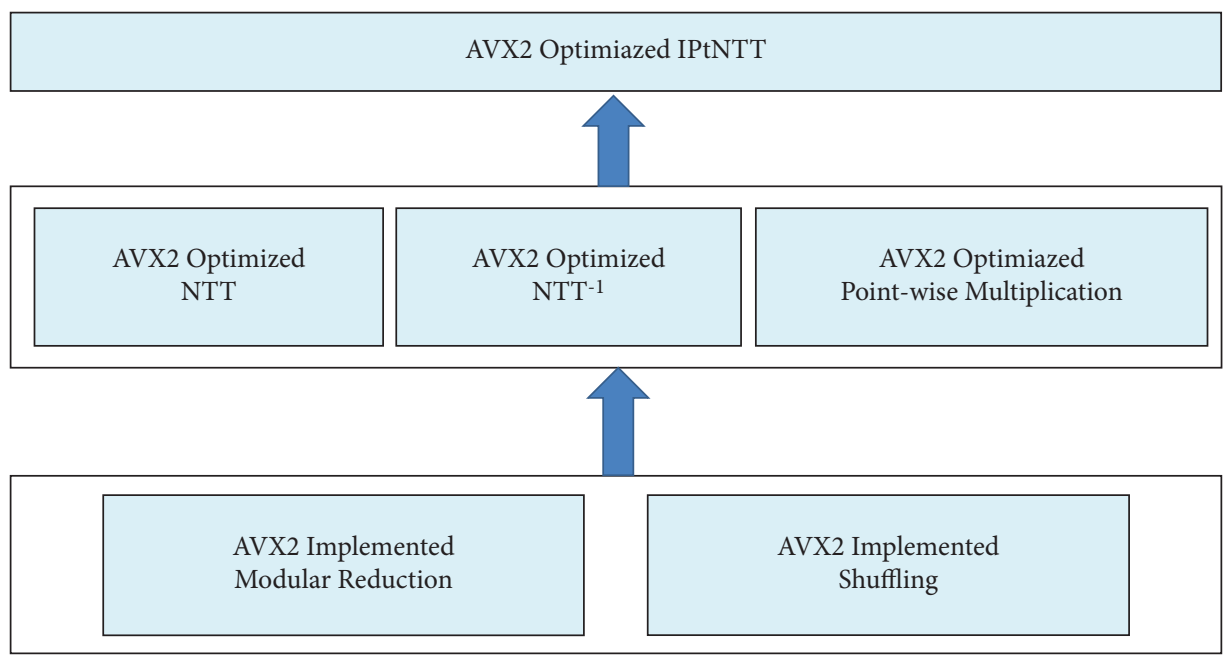

FIgUre 2: Modules of AVX2 optimized IPtNTT.

powers of the root, we multiply the brv(i)-th power of the root with $2^{16}$ and store the remainder of the product modulo $q$. Then, the Montgomery transformed product of any integer and the value in the table is within $(-q, q)$. The powers table of the inverse of the root used for the inverse transformation can also be constructed in this way. To ensure the correctness of the results, we additionally perform Barrett transformation on the results of each level. The procedures of the forward transformation NTT and the inverse transformation $\mathrm{NTT}^{-1}$ are presented in Algorithms 4 and 5, respectively.

When doing the point-wise multiplication, we only need to use AVX2 instructions to do the parallel computation. It should be noted that those multiplications which cannot be precomputed can be implemented through two Montgomery transformations. To achieve this, first Montgomery transform the product of a multiplier and $2^{32}(\bmod q)$, and then Montgomery transform the product of the result and the other multiplier.
3.4. AVX2 Implementation of IPtNTT. In order to improve the computation efficiency, we take advantages of some techniques in point-wise multiplication. The technical details of IPtNTT are given below.

From 2.2, we can see the time complexity of IPtNTT mainly includes two parts. One is the complexity of NTT and $\mathrm{NTT}^{-1}$ to the shorter polynomials, i.e., $\bar{f}_{i}(z)$ and $\bar{g}_{i}(z)$, the AVX2 implementation of which has been presented in 3.3. The other is the complexity of the point-wise multiplications mentioned in Step 2 of Algorithm 1. We deduce an applicable expression for arbitrary rounds of IPtNTT based on [8]. In the situation that we have acquired $\overline{\bar{f}}_{i}$ and $\widehat{\bar{g}}_{i}$, i.e., NTT transformed coefficients of $\bar{f}_{i}(z)$ and $\bar{g}_{i}(z)$, where $0 \leq i<2^{\alpha}$, we may acquire $\widehat{\bar{h}}_{i}$, the NTT coefficients of $\bar{h}_{i}$ as follows:

$$
\begin{aligned}
& i \equiv 0 \bmod 2: \\
& \widehat{\bar{h}}_{i}=\widehat{\bar{f}}_{\frac{2}{2}} \hat{\overline{\mathrm{g}}}_{\mathrm{i}}+\sum_{\mathrm{l}=0}^{\frac{\mathrm{i}}{2}-1}\left[\left(\hat{\overline{\mathrm{f}}}_{\mathrm{l}}+\hat{\overline{\mathrm{f}}}_{\mathrm{i}-1}\right) \circ\left(\hat{\overline{\mathrm{g}}}_{\mathrm{l}}+\widehat{\overline{\mathrm{g}}}_{\mathrm{i}-1}\right)-\hat{\overline{\mathrm{f}}}_{\mathrm{l}}^{\circ} \hat{\overline{\mathrm{g}}}_{\mathrm{l}}-\hat{\overline{\mathrm{f}}}_{\mathrm{i}-1} \circ \hat{\overline{\mathrm{g}}}_{\mathrm{i}-1}\right]+
\end{aligned}
$$

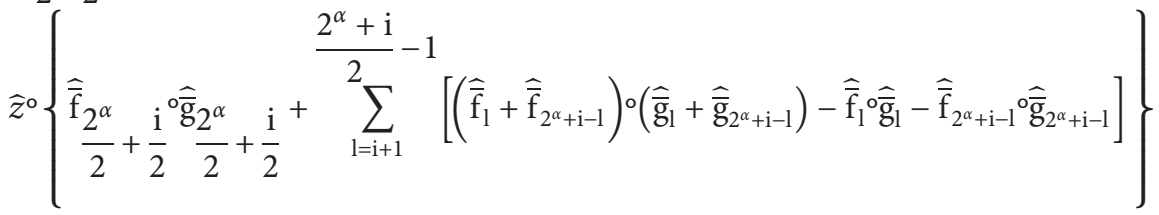

$$
\begin{aligned}
& i \equiv 1 \bmod 2 \text { : } \\
& \widehat{\bar{h}}_{i}=\sum_{l=0}^{i-1}\left[\left(\widehat{\bar{f}}_{l}+\widehat{\bar{f}}_{i-l}\right) \circ\left(\widehat{\overline{\mathrm{g}}}_{\mathrm{l}}+\widehat{\overline{\mathrm{g}}}_{\mathrm{i}-1}\right)-\widehat{\overline{\mathrm{f}}}_{\mathrm{l}} \circ \widehat{\overline{\mathrm{g}}}_{\mathrm{l}}-\widehat{\overline{\mathrm{f}}}_{\mathrm{i}-1} \circ \widehat{\overline{\mathrm{g}}}_{\mathrm{i}-1}\right]+ \\
& \frac{2^{\alpha}+\mathrm{i}-1}{2} \\
& \widehat{z}^{\circ} \sum_{l=i+1}^{2}\left[\left(\widehat{\overline{\mathrm{f}}}_{1}+\widehat{\overline{\mathrm{f}}}_{2^{\alpha}+\mathrm{i}-1}\right) \circ\left(\widehat{\overline{\mathrm{g}}}_{\mathrm{l}}+\widehat{\overline{\mathrm{g}}}_{2^{\alpha}+\mathrm{i}-1}\right)-\widehat{\overline{\mathrm{f}}}_{\mathrm{l}}^{\circ} \widehat{\overline{\mathrm{g}}}_{\mathrm{l}}-\widehat{\overline{\mathrm{f}}}_{2^{\alpha}+\mathrm{i}-1} \circ \widehat{\overline{\mathrm{g}}}_{2^{\alpha}+\mathrm{i}-1}\right],
\end{aligned}
$$


Input: An integer $a$ satisfies $-2^{15} q<a=2{ }^{16} a_{1}+a_{0}<2^{15} q$, where $0 \leq a_{0}<2^{16}, q<2^{15}$

Output: $r^{\prime}=2^{-16} a(\bmod q)$, where $-q<r^{\prime}<q$

Step 1: Using _mm256_mullo_epi16 $(*, * *)$, calculate and store the lower 16 bits of the product of two numbers;

Step 2: Using _mm256_mulhi_epi16 $(*, * *)$, calculate and store the higher 16 bits of the signed product of two numbers;

Step 3: Using_mm256_mullo_epi16 $\left(*, q^{-1}\right)$, compute and store the lower 16 bits of the product between the result of Step 1 and $q^{-1}$, which has been precomputed;

Step 4: Using _mm256_mulhi_epi16 $(*, q)$, calculate and store the higher 16 bits of the product of the result of Step 3 and $q$;

Step 5: Using_mm256_sub_epi16 $(*, * *)$, calculate and store the difference between the result of Step 2 and the result of Step 4.

Algorithm 2: Montgomery transformation.

Input: An integer $a$ satisfies $-2^{15}<a<2^{15}$, where $0 \leq q<2^{15}$

Output: $r=a(\bmod q)$, where $0 \leq r \leq q$

Step 1: Using_mm256_mulhi_epi16 $(a, v)$, calculate and store the high 16 bits of the signed product of $a$ and $v$, where $v$, which is the integer closest to $2^{\lfloor\log (q)\rfloor+15} / \bar{q}$, has been precomputed;

Step 2: Using_mm256_srai_epi16 $(*,(\lfloor\log (q)\rfloor-1))$, shift the result of Step 1 to the right for $(\lfloor\log (q)\rfloor-1)$ bits;

Step 3: Using_mm256_mullo_epi16 $(*, q)$, calculate and store the lower 16 bits of the product of the result of Step 2 and $q$;

Step 4: Using_mm256_sub_epi16 $(a, *)$, calculate and store the difference between $a$ and the result of Step 3.

Algorithm 3: Barrett transformation.

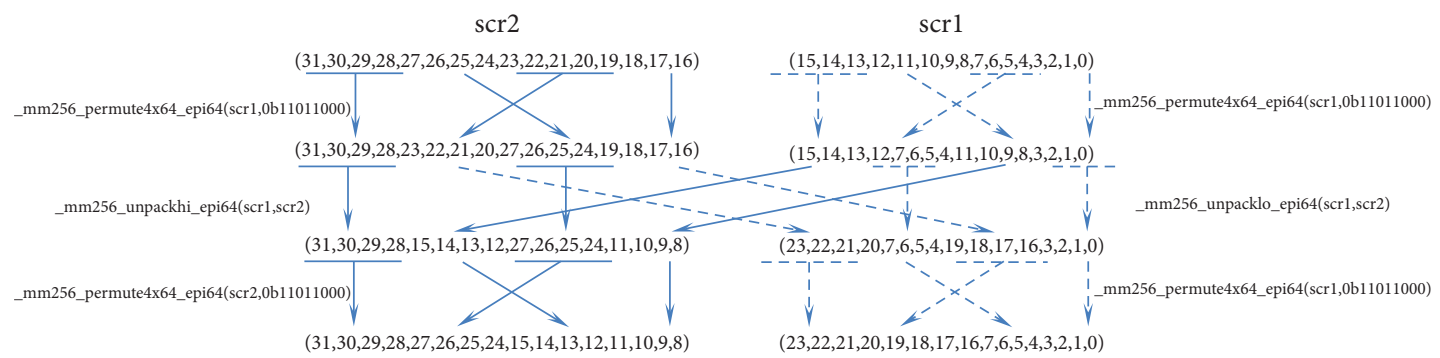

Figure 3: Shuffle in the fourth level from the bottom in NTT, where scr1 and scr2 are two vectors.

Input: Coefficient vector of $f$, which is $\left(f_{0}, f_{1}, \ldots, f_{n-1}\right)$

Output: Bit-reversed NTT coefficient vector of $f$, which is $\left(\hat{f}_{\operatorname{brv}(0)}, \widehat{f}_{\operatorname{brv}(1)}, \ldots, \hat{f}_{\operatorname{brv}(n-1)}\right)$

Step 1: Using_mm256_set1_epi16 (*), or_mm256_set_epi16 for the last 4 levels, store the value from the powers table of the root used in this round;

Step 2: Using _mm256_load_si256 (*), store the coefficient vectors;

Step 3: Shuffle if it is in the last 4 levels, skip this otherwise;

Step 4: Montgomery transform the products of higher degree coefficients and the result of Step 1;

Step 5: Using_mm256_sub_epi16 $(*, * *)$, compute the difference between the lower degree coefficients and the result of Step 4 to the higher degree coefficient vector;

Step 6: Using_mm256_add_epi16 $(*, * *)$, compute the sum of the lower degree coefficients and the result of Step 4 to the lower degree coefficient vector;

Step 7: Barrett transform the results of Step 5 and Step 6, respectively;

Step 8: Restore the coefficients if it is in the last 4 levels, skip this otherwise;

Step 9: Using _mm256_store_si256 (*), store the result of the last step;

Step 10: Circulate from Step 2, until the value stored in Step 1 needs to be updated;

Step 11: Circulate from Step 1, until all coefficients have been transformed;

Step 12: Enter the next level, repeat steps above till the last level. 
Input: Bit-reversed NTT coefficient vector of $f$, which is $\left(\hat{f}_{\operatorname{brv}(0)}, \hat{f}_{\operatorname{brv}(1)}, \ldots, \hat{f}_{\operatorname{brv}(n-1)}\right)$

Output: Coefficient vector of $f$, which is $\left(f_{0}, f_{1}, \ldots, f_{n-1}\right)$

Step 1: Using_mm256_set1_epi16 (*), or_mm256_set_epi16 for the first 4 levels, store the value from the powers table of the root used in this round;

Step 2: Using _mm256_load_si256 (*), store the coefficient vectors;

Step 3: Shuffle if it is in the last 4 levels, skip this otherwise;

Step 4: Using_mm256_add_epi16 $(*, * *)$, compute the sum of the two coefficient vectors from Step 3 or Step 2 to the lower degree coefficient vector;

Step 5: Using_mm256_sub_epi16 $(*, * *)$, compute the difference of the lower degree coefficient vector and the higher one from Step 3 or Step 2;

Step 6: Montgomery transform the products of the higher degree coefficients and the result of Step 1 and assign them to the higher degree coefficient vector;

Step 7: Barrett transform the result of Step 4 and Step 6, respectively;

Step 8: Restore the coefficients if it is in the first 4 levels, skip this otherwise;

Step 9: Using _mm256_store_si256 (*), store the result of the last step;

Step 10: Circulate from Step 2, until the value stored in Step 1 needs to be updated;

Step 11: Circulate from Step 1, until all coefficients have been transformed;

Step 12: Enter the next level, repeat steps above till the last level.

Step 13: Montgomery transform the products of the result of Step 12 and $2^{16} n^{-1}(\bmod q)$, then do the Barrett transformation.

Algorithm 5: Implementation of $\mathrm{NTT}^{-1}$.

TABLE 1: $T(n)$ of IPtNTT with different $n$ and $q$.

\begin{tabular}{lccc}
\hline$\alpha$ & $n=256$ & $n=512$ & $n=1024$ \\
\hline 0 & 6400 & 14336 & 31744 \\
1 & 5888 & 13312 & 29696 \\
2 & 5440 & 12416 & 27904 \\
3 & 5216 & 11968 & 27008 \\
4 & 5488 & 12512 & 28096 \\
5 & 6776 & 15088 & 33248 \\
6 & 10108 & 21752 & 46576 \\
7 & 17534 & 36604 & 76280 \\
8 & 33151 & 67838 & 138748 \\
9 & 65151.5 & 131839 & 266750 \\
\hline
\end{tabular}

Input: $\widehat{\bar{f}}_{i}$ and $\hat{\bar{g}}_{i}$, NTT coefficient vectors of $\bar{f}_{i}(z)$ and $\bar{g}_{i}(z)$, where $0 \leq i<2^{\alpha}$

Output: $\widehat{\bar{h}}_{i}$, NTT coefficient vector of $\bar{h}_{i}$

Step 1: Montgomery transform the product of $\hat{\bar{f}}_{i}$ and $2^{32}(\bmod q)$;

Step 2: Montgomery transform the result of $\overline{\bar{f}}_{i}{ }^{\circ} \overline{\mathrm{g}}_{\mathrm{i}}$ and $\left(\widehat{\bar{f}}_{i}+\overline{\bar{f}}_{\mathrm{j}}\right)^{\circ}\left(\overline{\overline{\mathrm{g}}}_{\mathrm{i}}+\widehat{\overline{\mathrm{g}}}_{\mathrm{j}}\right)$, where $\hat{\bar{f}}_{i}$ is replaced by the result of Step 1;

Step 3: Compute and Barrett transform the part in parentheses which will perform point-wise multiplication with $\widehat{z}$ in equation (6);

Step 4: Montgomery transform $\widehat{z}^{\circ}(*)$ in equation (6);

Step 5: Compute and Barrett transform the remaining additions and subtractions in equation (6).

Algorithm 6: Implementation of point-wise multiplication in IPtNTT.

It is clear that $\hat{\bar{f}}_{i} \circ \hat{\overline{\mathrm{g}}}_{\mathrm{i}}$ and $\left(\hat{\bar{f}}_{i}+\hat{\bar{f}}_{j}\right) \circ\left(\hat{\overline{\mathrm{g}}}_{\mathrm{i}}+\hat{\overline{\mathrm{g}}}_{\mathrm{j}}\right)$, where $0 \leq i, j<2^{\alpha}{ }_{2}$ can be used over and over again once computed to acquire $\bar{h}_{i}$, so as to reduce the point-wise multiplications. Since the whole process includes $2^{\alpha}$ multiplications like $\hat{\bar{f}}_{i}{ }^{\circ} \overline{\bar{g}}_{\mathrm{i}}, C_{2^{\alpha}}^{2}$ multiplications like $\left(\hat{\bar{f}}_{i}+\widehat{\bar{f}}_{j}\right)^{\circ}\left(\hat{\overline{\mathrm{g}}}_{\mathrm{i}}+\widehat{\overline{\mathrm{g}}}_{\mathrm{j}}\right)$, and $2^{\alpha}-1$ multiplications like $\widehat{z}^{\circ}(*), \quad \alpha$ IPtNTT includes $2^{2 \alpha-1}+3 \cdot 2^{\alpha-1}-1$ point-wise multiplications in total. Including $2^{\alpha+1} \mathrm{NTTs}$ and $2^{\alpha} \mathrm{NTT}^{-1} \mathrm{~s}$, the computational complexity of $\alpha \mathrm{IPtNTT}$ is as follows:

$$
\begin{aligned}
T(n) & =\left(2^{2 \alpha-1}+3 \cdot 2^{\alpha-1}-1\right) \cdot \frac{n}{2^{\alpha}}+2^{\alpha+1} \cdot \frac{n}{2^{\alpha}} \log \frac{n}{2^{\alpha}}+2^{\alpha} \cdot \frac{n}{2^{\alpha}} \log \frac{n}{2^{\alpha}} \\
& =2^{\alpha-1} n+\frac{3}{2} n-\frac{n}{2^{\alpha}}-3 \alpha n+3 n \log n .
\end{aligned}
$$

Let $\alpha$ traverse $\mathbb{Z}_{10}$, and we compute $T(n)$ when $n=256,512,1024$, and present the result in Table 1 . Note that $\widehat{z}$ can also be precomputed as $\widehat{z}=\operatorname{NTT}(0,1,0, \ldots, 0)$, 


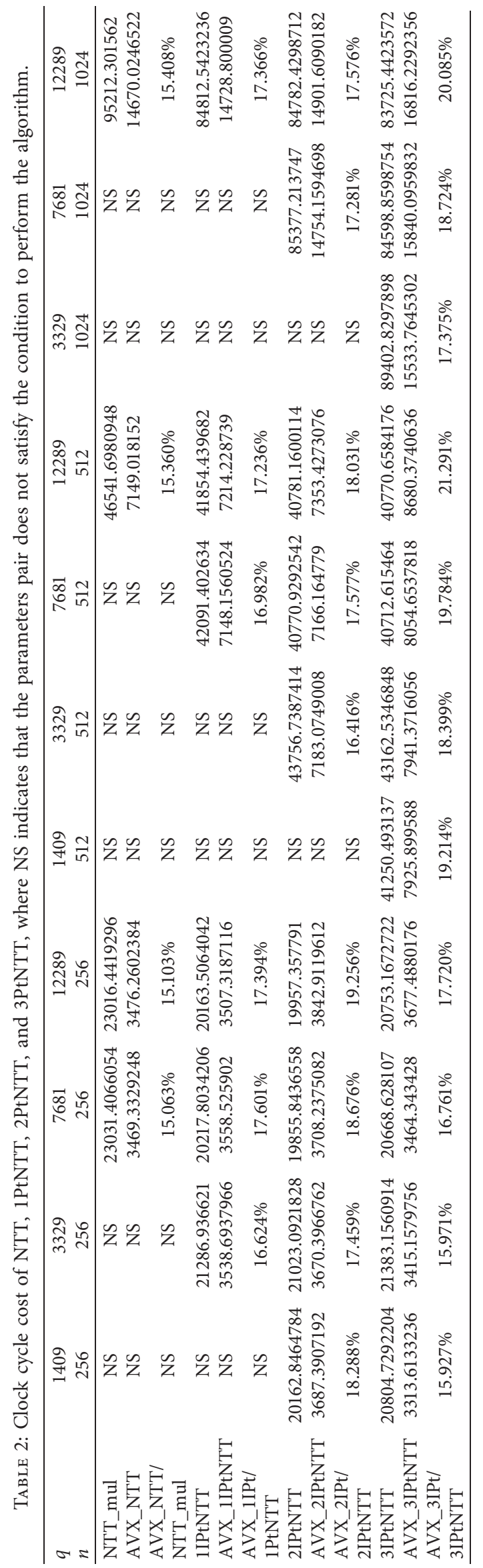


and we store the remainder of the product of $\widehat{z}$ and $2^{16}$ modulo $q$ for the convenience of Montgomery transformation. The AVX2 implementation equation (6) can be described as Algorithm 6.

Considering the limitation to the input, we need to do different times of Barrett transformations as the chosen $q$ differs. For example, if $q=12289$, we need to Barrett transform after every addition or subtraction; if $q=7681$, we need to Barrett transform after every three additions or subtractions; if $q=3329$ or even less, we do not need to do extra Barrett transformations when $\alpha \leq 3$.

\section{Experimental Results and Analysis}

We obtained the results of computational cost experiments of $\mathrm{C}$ implemented multiplication on polynomial rings by using NTT, 1IPtNTT, 2IPtNTT, and 3IPtNTT, which are marked as NTT_mul and $\alpha$ IPtNTT_mul, and their AVX2 optimization, which are marked as AVX2_NTT and AVX2_ $\alpha$ IPtNTT, by running the implementation on a $2.5 \mathrm{GHZ}$ Intel Core i5 processor. We compiled the C implementation and AVX2 optimization with gcc 7.5.0 on Ubuntu 18.04 with the core Linux 4.15.0. We chose several pairs of $(n, q)$ in our experiments. For each pair, we performed 10 rounds of experiments with $2^{16}$ times of tests in each round and compute the average cost of 1 test. We chose 1409, 3329, 7681, and 12289 for $q$ and 256, 512, and 1024 for $n$, respectively. We present our experimental results in Table 2.

Analyzing Table 2, we may confirm that the computational cost of NTT, 1IPtNTT, 2IPtNTT, and 3IPtNTT after AVX2 optimization is reduced to $15 \%-22 \%$. Thus, for certain $q$ and $n$, when $n=512,1024$, we suggest implementing AVX_ $\alpha$ IPtNTT, where $\alpha=\min \{r: q \equiv 1(\bmod$ $\left.\left.\left(n / 2^{r-1}\right)\right), r \geq 0\right\}$. For example, if $q=3329, n=512$, we suggest using AVX_2IPtNTT; if $q=7681, n=512$, we suggest using AVX_1IPtNTT; if $q=12289, n=512,1024$, we suggest using AVX_NTT. When $n=256$, AVX_3IPtNTT is the best choice if $q$ is within 13 bits; if $q=12289$, AVX_NTT is the most efficient.

\section{Conclusion}

IPtNTT is a method to fast realize multiplication on polynomial rings. Due to its significant advantage in expanding the parameter selection range of lattice public-key cryptographic schemes, it can be widely used in the programming of lattice public-key cryptographic schemes. In this paper, we present the AVX2 optimization implementation method of IPtNTT and show the experimental results with different parameters, based on the AVX2 implementation of technique modules such as modular reduction, coefficients shuffling, and point-wise multiplication of coefficient vectors. According to the experimental results, the implementation suggestions on using IPtNTT to complete the multiplication on polynomial rings are given. The research results in this paper can be applied to the fast implementation of lattice-based public-key cryptography schemes.

\section{Data Availability}

The AVX2 implementation of our work is available at https://github.com/ghygit/AVX2-optimized-3IPtNTT.git.

\section{Conflicts of Interest}

The authors declare that there are no conflicts of interest.

\section{Acknowledgments}

This work was supported by the National Science Foundation (grant nos. 61702548 and 61902428).

\section{References}

[1] O. Regev, "On lattices, learning with errors, random linear codes, and cryptography," Journal of the ACM, vol. 56, no. 6, pp. 1-40, 2009.

[2] V. Lyubashevsky, C. Peikert, and O. Regev, "On ideal lattices and learning with errors over rings," in Proceedings of the Annual International Conference on the Theory and Applications of Cryptographic Techniques, pp. 1-23, Springer, Monaco and Nice, France, May 2010.

[3] A. Langlois and D. Stehlé, "Worst-case to average-case reductions for module lattices," Designs, Codes and Cryptography, vol. 75, no. 3, pp. 565-599, 2015.

[4] A. Karatsuba, "Multiplication of multidigit numbers on automata," Soviet physics doklady, vol. 7, pp. 595-596, 1963.

[5] A. A. Karatsuba and Y. P. Ofman, "Multiplication of manydigital numbers by automatic computers," Russian Academy of Sciences, vol. 145, no. 2, pp. 293-294, 1962.

[6] J. W. Cooley and J. W. Tukey, "An algorithm for the machine calculation of complex Fourier series," Mathematics of Computation, vol. 19, no. 90, pp. 297-301, 1965.

[7] S. Zhou, H. Xue, D. Zhang et al., "Preprocess-then-NTT technique and its applications to kyber and new hope," in Proceedings of the International Conference on Information Security and Cryptology, pp. 117-137, Springer, Seoul, Korea, November 2018.

[8] Y. Zhu, Z. Liu, and Y. Pan, "When NTT meets Karatsuba: preprocess-then-NTT technique revisited," IACR Cryptology ePrint Archieve, vol. 20191079 pages, 2019.

[9] G. Seiler, Faster AVX2 Optimized NTT Multiplication for Ring-LWE Lattice cryptographyIBM Research, Zurich. Switzerland, 2018.

[10] V. Lyubashevsky, D. Micciancio, C. Peikert, and A. Rosen, "SWIFFT: a modest proposal for FFT hashing," in Proceedings of the International workshop on fast software encryption, pp. 54-72, Springer, Lausanne, Switzerland, February 2008.

[11] E. Chu and A. George, Inside the FFT Black Box: Serial and Parallel Fast Fourier Transform algorithms, CRC Press, Boca Raton, FL, USA, 1999. 